\title{
Optimal Portfolio of Corporate Investment and Consumption Problem under Market Closure: Inflation Case
}

\author{
Zongyuan Huang ${ }^{1}$ and Detao Zhang ${ }^{2}$ \\ ${ }^{1}$ School of Mathematics, Shandong University, Jinan 250100, China \\ ${ }^{2}$ School of Economics, Shandong University, Jinan 250100, China \\ Correspondence should be addressed to Detao Zhang; zhangdetao@sdu.edu.cn
}

Received 21 January 2013; Revised 24 March 2013; Accepted 22 April 2013

Academic Editor: Guangchen Wang

Copyright ( 2013 Z. Huang and D. Zhang. This is an open access article distributed under the Creative Commons Attribution License, which permits unrestricted use, distribution, and reproduction in any medium, provided the original work is properly cited.

\begin{abstract}
We present the model of corporate optimal investment with consideration of the influence of inflation and the difference between the market opening and market closure. In our model, the investor has three market activities of his or her choice: investment in project $\mathrm{A}$, investment in project $\mathrm{B}$, and consumption. The optimal strategy for the investor is obtained using the HamiltonJacobi-Bellman equation which is derived using the dynamic programming principle. Further along, a specific case, the Hyperbolic Absolute Risk Aversion case, is discussed in detail, where the explicit optimal strategy can be obtained using a very simple and direct method. At the very end, we present some simulation results along with a brief analysis of the relationship between the optimal strategy and other factors.
\end{abstract}

\section{Introduction}

Inflation originally referred to the debasement of the currency. When the government increases the total number of the currency, the relative value of the currency decreases. As a result, consumers would need more money to exchange for the same goods and services. The term inflation usually refers to a measured rise in a broad price index that represents the overall level of prices in goods and services in the economy. The Consumer Price Index (CPI), the Personal Consumption Expenditures Price Index (PCEPI), and the GDP deflator are some examples of broad price indices. These factors play an important role in economics, because of which they are studied in the theory.

Portfolio diversification plays an important role in finance. In Merton [1] and Duffie [2], a stochastic model is first given to analyze the security market, while Karatzas [3], based on the stochastic analysis, presented the model considering the split of consumption and investment of the investors. Choi [4], on the other hand, paid attention to the international corporate investment where investments were made into real corporate projects. Afterwards, Bellalah and $\mathrm{Wu}[5]$ extended the theory of corporate international investment from Choi into an environment with the presence of incomplete information and taxation from governments. Their model was concerned with the international diversification problem in finance and gave reasonable analysis to the "home bias puzzle." In 2006, a model giving the optimal corporate portfolio and consumption choice was presented by $\mathrm{Wu}$ and Zhang [6], while Bellalah and $\mathrm{Wu}$ [7] modeled the market closure together with the international securities portfolio management with incomplete information.

In our paper, we present a model of corporate investment and consumption choice problem with market closure in inflation case, so that we can derive the optimal investment strategy. Our model is an extension of the usual corporate investment model from Bellalah and $\mathrm{Wu}[5,7], \mathrm{Wu}$ and Zhang [6], Huang and Wu [8], and D. T. Zhang and T. Zhang [9] since we take into consideration the influence of inflation and the investment on two projects.

In this model, we suppose that the investor has an option to invest his money into two different projects, $\mathrm{A}$ and $\mathrm{B}$. In real life, the production of the factory may be different between the day and the night; therefore, in our model, we have equations describing the input price and the output price of the production in the daytime and night with specified 
instantaneous expected rates and instantaneous volatilities. Also, we develop two pieces of production equations corresponding to the daytime and nighttime production which is a function of the output price. With the input and output price model and the production quantity model, we can easily derive the expression for the net profit of the corporate investment after considering taxation of the government.

Finally, instead of making investment, the investor has the option to make consumption for satisfaction as well.

During the nighttime, banking activities will not be allowed; therefore, the investment portfolio will be the same throughout the night. However, the investor is still allowed to consume to increase utility. Clearly, the consumption in the daytime and the night will be different.

In general, we consider corporate investment and consumption altogether to maximize the utility of wealth for the investor aiming to find out the optimal strategy for the investment. For the general model, it is difficult to solve for the optimal value function explicitly. Therefore, we considered a specific and important case of utility function, Hyperbolic Absolute Risk Aversion (HARA) case, to get the optimal solution. It turns out that we can write out the explicit optimal solution using a very simple and direct method which will be discussed in Section 3 of the paper.

In the next section, we will present the model and the assumptions of the optimal investment problem. In the HARA cases, besides giving the explicit optimal solution of the corporate investment model using dynamic programming principle, we will give analysis of the model in the economical point of view in Section 3.

At the end of this paper, some simulation results will be given to provide a more numerical interpretation of the model. Relationship between the optimal portfolio and important factors such as the volatility and the interest rate will be given together with computer-drawn graphs.

\section{Model and Formulation of the Problem}

Let $(\Omega, \mathscr{F}, P)$ be a complete probability space endowed with a filtration $\left\{\mathscr{F}_{t}: 0 \leq t \leq T\right\} .\left\{W_{t}\right\}_{0 \leq t \leq T},\left\{B_{t}^{1}\right\}_{0 \leq t \leq T}$, and $\left\{B_{t}^{2}\right\}_{0 \leq t \leq T}$ are three 1-dimensional Brownian motions defined in this space, which represent the external sources of uncertainty in the market, and the correlation coefficients between them are $\rho_{1}, \rho_{2}, \rho_{1,2}$ with $-1 \leq \rho_{1}, \rho_{2}, \rho_{1,2} \leq 1$.

Denote the rate of inflation by $I_{t}$, which is stochastic and depends on the situation of the economic. Like some pricing processes of the stochastic assets, we use a geometric Brownian motion to describe the inflation rate level at time $t$, that is,

$$
d I_{t}=I_{t} \theta_{t} d t+I_{t} \eta_{t} d W_{t}
$$

The drift of the process $\theta$ is the expected rate of inflation per unit of time, and it is defined by

$$
\theta_{t}=\lim _{\delta \downarrow 0} E\left(\frac{I_{t+\delta}-I_{t}}{\delta I_{t}}\right),
$$

where $(\eta)^{2}$ is the variance of the process per unit of time defined by

$$
\left(\eta_{t}\right)^{2}=\lim _{\delta \downarrow 0} E\left[\frac{1}{\delta}\left(\frac{I_{t+\delta}-I_{t}}{I_{t}}-\delta \theta_{t}\right)^{2}\right] .
$$

Without loss of generality, we assume that $I_{0}=1$; that is, there is no inflation at initial time $t=0$.

In this model, we suppose that the investor can choose two real projects, $\mathrm{A}$ and $\mathrm{B}$, with some production to get a higher return but with certain risk. The input price and output price are not the same during the day and the night. The duration of the production in the daytime is $T$, and the duration at the nighttime is $N$. Then the daytime is $[0, T]$, $[T+N, 2 T+N], \ldots,[(n-1)(T+N), n T+(n-1) N]$, denoted by $D$, while the nighttime is $[T, T+N],[2 T+N, 2 T+2 N]$, $\ldots,\left[(n T+(n-1) N, n(T+N)]\right.$, denoted by $D^{C}$.

For project A, we denote $P(t)$ and $\bar{P}(t)$ as the the input prices in the daytime and nighttime, respectively. $S(t)$ and $\bar{S}(t)$ are the output prices, while $Q(t)$ and $\bar{Q}(t)$ are the quantity of the production and $R(t)$, and $\bar{R}(t)$ are the cash flow.

In economic theory, the quantity of the production is the function of the input price, $Q(t)=[S(t)]^{\beta_{1}}$, where $\beta_{1}<0$ in general. The cash flow of project $A$ in daytime and nighttime are as follows:

$$
\begin{array}{r}
R(t)=(1-\tau)[S(t)-P(t)] Q(t), \\
Q(t)=[S(t)]^{\beta_{1}}, \quad t \in D, \\
\bar{R}(t)=(1-\tau)[\bar{S}(t)-\bar{P}(t)] \bar{Q}(t), \\
\bar{Q}(t)=[\bar{S}(t)]^{\beta_{2}}, \quad t \in D^{C} .
\end{array}
$$

Similarly, we can define the corresponding variables of project B using the letters which have the symbol *. And the cash flow of project B in daytime and nighttime are as follows:

$$
\begin{array}{r}
R^{*}(t)=(1-\tau)\left[S^{*}(t)-P^{*}(t)\right] Q^{*}(t), \\
Q^{*}(t)=\left[S^{*}(t)\right]^{\beta_{1}^{*}}, \quad t \in D, \\
\bar{R}^{*}(t)=(1-\tau)\left[\bar{S}^{*}(t)-\bar{P}^{*}(t)\right] \bar{Q}^{*}(t), \\
\bar{Q}^{*}(t)=\left[\bar{S}^{*}(t)\right]^{\beta_{2}^{*}}, \quad t \in D^{C} .
\end{array}
$$

We suppose the input price and output price satisfy the following equation:

$$
\begin{gathered}
d P(t)=\alpha_{P}(t) P(t) d t+\sigma(t) P(t) d B_{t}^{1}, \quad t \in D, \\
d \bar{P}(t)=\bar{\alpha}_{P}(t) \bar{P}(t) d t+\bar{\sigma}(t) \bar{P}(t) d B_{t}^{1}, \quad t \in D^{C}, \\
d S(t)=\alpha_{S}(t) S(t) d t+\sigma(t) S(t) d B_{t}^{1}, \quad t \in D, \\
d \bar{S}(t)=\bar{\alpha}_{S}(t) \bar{S}(t) d t+\bar{\sigma}(t) \bar{S}(t) d B_{t}^{1}, \quad t \in D^{C}, \\
d P^{*}(t)=\alpha_{P}^{*}(t) P^{*}(t) d t+\sigma^{*}(t) P^{*}(t) d B_{t}^{2}, \quad t \in D, \\
d \bar{P}^{*}(t)=\bar{\alpha}_{P}^{*}(t) \bar{P}^{*}(t) d t+\bar{\sigma}^{*}(t) \bar{P}^{*}(t) d B_{t}^{2}, \quad t \in D^{C}, \\
d S^{*}(t)=\alpha_{S}^{*}(t) S^{*}(t) d t+\sigma^{*}(t) S^{*}(t) d B_{t}^{2}, \quad t \in D, \\
d \bar{S}^{*}(t)=\bar{\alpha}_{S}^{*}(t) \bar{S}^{*}(t) d t+\bar{\sigma}^{*}(t) \bar{S}^{*}(t) d B_{t}^{2}, \quad t \in D^{C},
\end{gathered}
$$


where $P(t)$ and $S(t)$ have initial values $P_{0}, S_{0}$, respectively. Here $\alpha_{P}, \bar{\alpha}_{P}$ represent the instantaneous expected input rates in the day and the night, respectively, while $\alpha_{S}, \bar{\alpha}_{S}$ represent the instantaneous expected output rates. $\sigma, \bar{\sigma}$ are instantaneous volatilities in the day and the night, respectively.

According to Ito's formula, we know that the cash flow for project $\mathrm{A}$ and $\mathrm{B}$ satisfy the following equations:

$$
\begin{aligned}
d R(t) & =R(t)\left[f(t) d t+\left(1+\beta_{1}\right) \sigma(t) d B_{t}^{1}\right], \quad t \in D, \\
d \bar{R}(t) & =\bar{R}(t)\left[\bar{f}(t) d t+\left(1+\beta_{2}\right) \bar{\sigma}(t) d B_{t}^{1}\right], \quad t \in D^{C},
\end{aligned}
$$

where

$$
\begin{aligned}
& f(t)=\frac{1}{2}\left(1+\beta_{1}\right)^{2} \sigma^{2}+\frac{F^{\prime}(t)}{F(t)}, \\
& \bar{f}(t)=\frac{1}{2}\left(1+\beta_{2}\right)^{2} \bar{\sigma}^{2}+\frac{\bar{F}^{\prime}(t)}{\bar{F}(t)},
\end{aligned}
$$

$d R^{*}(t)=R^{*}(t)\left[f^{*}(t) d t+\left(1+\beta_{1}^{*}\right) \sigma^{*}(t) d B_{t}^{2}\right], \quad t \in D$, $d \bar{R}^{*}(t)=\bar{R}^{*}(t)\left[\bar{f}^{*}(t) d t+\left(1+\beta_{2}^{*}\right) \bar{\sigma}^{*}(t) d B_{t}^{2}\right], \quad t \in D^{C}$,

where

$$
\begin{aligned}
& f(t)=\frac{1}{2}\left(1+\beta_{1}\right)^{2} \sigma^{2}+\frac{F^{\prime}(t)}{F(t)}, \\
& \bar{f}(t)=\frac{1}{2}\left(1+\beta_{2}\right)^{2} \bar{\sigma}^{2}+\frac{\bar{F}^{\prime}(t)}{\bar{F}(t)}, \\
& f^{*}(t)=\frac{1}{2}\left(1+\beta_{1}^{*}\right)^{2}\left(\sigma^{*}\right)^{2}+\frac{\left(F^{*}\right)^{\prime}(t)}{F^{*}(t)}, \\
& \bar{f}^{*}(t)=\frac{1}{2}\left(1+\beta_{2}^{*}\right)^{2}\left(\bar{\sigma}^{*}\right)^{2}+\frac{\left(\bar{F}^{*}\right)^{\prime}(t)}{\bar{F}^{*}(t)},
\end{aligned}
$$

with

$$
\begin{aligned}
F(t)=\{ & S((k-1)(T+N)) \\
& \times \exp \left[\int_{(k-1)(T+N)}^{t}\left(\alpha_{S}(r)-\frac{1}{2} \sigma^{2}(r)\right) d r\right] \\
& -P((k-1)(T+N)) \\
& \left.\times \exp \left[\int_{(k-1)(T+N)}^{t}\left(\alpha_{P}(r)-\frac{1}{2} \sigma^{2}(r)\right) d r\right]\right\} \\
\times & \exp \left[\beta_{1} \int_{(k-1)(T+N)}^{t}\left(\alpha_{S}(r)-\frac{1}{2} \sigma^{2}(r)\right) d r\right], \\
\bar{F}(t)= & \bar{S}(k T+(k-1) N) \\
& \quad \times \exp \left[\int_{k T+(k-1) N}^{t}\left(\bar{\alpha}_{S}(r)-\frac{1}{2} \bar{\sigma}^{2}(r)\right) d r\right] \\
& \quad-P(k T+(k-1) N)
\end{aligned}
$$

$$
\begin{gathered}
\left.\times \exp \left[\int_{k T+(k-1) N}^{t}\left(\bar{\alpha}_{P}(r)-\frac{1}{2} \sigma^{2}(r)\right) d r\right]\right\} \\
\times \exp \left[\beta_{2} \int_{k T+(k-1) N}^{t}\left(\bar{\alpha}_{S}(r)-\frac{1}{2} \sigma^{2}(r)\right) d r\right] . \\
F^{*}(t)=\cdots, \quad \bar{F}^{*}(t)=\cdots .
\end{gathered}
$$

Similarly, we can get $F^{*}(t)$ and $\bar{F}^{*}(t)$ just substitute $\alpha^{*}\left(\sigma^{*}\right)$ for $\alpha(\sigma)$ and substitute $\bar{\alpha}^{*}\left(\bar{\sigma}^{*}\right)$ for $\bar{\alpha}(\bar{\sigma})$.

Since the existence of the inflation, the actual value of the input price should be $U_{t}=P_{t} / I_{t}$. Notice that the proportional rate of change of the real return of input price and output price should be described by

$$
\begin{aligned}
& d U(t)=U(t)\left[\left(\alpha_{P}(t)-\theta_{t}+\left(\eta_{t}\right)^{2}-\rho_{1} \sigma(t) \eta_{t}\right) d t\right. \\
& \left.+\sigma(t) d B_{t}^{1}-\eta_{t} d W_{t}\right], \quad t \in D \\
& d \bar{U}(t)=\bar{U}(t)\left[\left(\bar{\alpha}_{P}(t)-\theta_{t}+\left(\eta_{t}\right)^{2}-\rho_{1} \bar{\sigma}(t) \eta_{t}\right) d t\right. \\
& \left.+\bar{\sigma}(t) d B_{t}^{1}-\eta_{t} d W_{t}\right], \quad t \in D^{C}, \\
& d V(t)=V(t)\left[\left(\alpha_{S}(t)-\theta_{t}+\left(\eta_{t}\right)^{2}-\rho_{1} \sigma(t) \eta_{t}\right) d t\right. \\
& \left.+\sigma(t) d B_{t}^{1}-\eta_{t} d W_{t}\right], \quad t \in D, \\
& d \bar{V}(t)=\bar{V}(t)\left[\left(\bar{\alpha}_{S}(t)-\theta_{t}+\left(\eta_{t}\right)^{2}-\rho_{1} \bar{\sigma}(t) \eta_{t}\right) d t\right. \\
& \left.+\bar{\sigma}(t) d B_{t}^{1}-\eta_{t} d W_{t}\right], \quad t \in D^{C}, \\
& d U^{*}(t)=U^{*}(t)\left[\left(\alpha_{P}^{*}(t)-\theta_{t}+\left(\eta_{t}\right)^{2}-\rho_{2} \sigma^{*}(t) \eta_{t}\right) d t\right. \\
& \left.+\sigma^{*}(t) d B_{t}^{2}-\eta_{t} d W_{t}\right], \quad t \in D, \\
& d \bar{U}^{*}(t)=\bar{U}^{*}(t)\left[\left(\bar{\alpha}_{P}^{*}(t)-\theta_{t}+\left(\eta_{t}\right)^{2}-\rho_{2} \bar{\sigma}^{*}(t) \eta_{t}\right) d t\right. \\
& \left.+\bar{\sigma}^{*}(t) d B_{t}^{2}-\eta_{t} d W_{t}\right], \quad t \in D^{C}, \\
& d V^{*}(t)=V^{*}(t)\left[\left(\alpha_{S}^{*}(t)-\theta_{t}+\left(\eta_{t}\right)^{2}-\rho_{2} \sigma^{*}(t) \eta_{t}\right) d t\right. \\
& \left.+\sigma^{*}(t) d B_{t}^{2}-\eta_{t} d W_{t}\right], \quad t \in D, \\
& d \bar{V}^{*}(t)=\bar{V}^{*}(t)\left[\bar{\alpha}_{S}^{*}(t)-\theta_{t}+\left(\eta_{t}\right)^{2}-\rho_{2} \bar{\sigma}^{*}(t) \eta_{t} d t\right. \\
& \left.+\bar{\sigma}^{*}(t) d B_{t}^{2}-\eta_{t} d W_{t}\right], \quad t \in D^{C} .
\end{aligned}
$$

Finally the real cash flow for project $\mathrm{A}$ and $\mathrm{B}, \Lambda(t)=$ $R(t) / I(t), \bar{\Lambda}(t), \ldots$, satisfy

$$
\begin{array}{r}
d \Lambda(t)=\Lambda(t)\left\{\left[f(t)-\theta_{t}+\left(\eta_{t}\right)^{2}-\left(1+\beta_{1}\right) \rho_{1} \sigma(t) \eta_{t}\right] d t\right. \\
\left.+\left(1+\beta_{1}\right) \sigma(t) d B_{t}^{1}-\eta_{t} d W_{t}\right\}, \quad t \in D, \\
d \bar{\Lambda}(t)=\bar{\Lambda}(t)\left\{\left[\bar{f}(t)-\theta_{t}+\left(\eta_{t}\right)^{2}-\left(1+\beta_{2}\right) \rho_{1} \bar{\sigma}(t) \eta_{t}\right] d t\right. \\
\left.+\left(1+\beta_{2}\right) \bar{\sigma}(t) d B_{t}^{1}-\eta_{t} d W_{t}\right\}, \quad t \in D^{C}
\end{array}
$$




$$
\begin{aligned}
& d \Lambda^{*}(t) \\
& =\Lambda^{*}(t)\left\{\left[f^{*}(t)-\theta_{t}+\left(\eta_{t}\right)^{2}-\left(1+\beta_{1}^{*}\right) \rho_{2} \sigma^{*}(t) \eta_{t}\right] d t\right. \\
& \left.\quad+\left(1+\beta_{1}^{*}\right) \sigma^{*}(t) d B_{t}^{2}-\eta_{t} d W_{t}\right\}, \quad t \in D,
\end{aligned}
$$

$$
\begin{aligned}
& d \bar{\Lambda}^{*}(t) \\
& =\bar{\Lambda}^{*}(t)\left\{\left[\bar{f}^{*}(t)-\theta_{t}+\left(\eta_{t}\right)^{2}-\left(1+\beta_{2}^{*}\right) \rho_{2} \bar{\sigma}^{*}(t) \eta_{t}\right] d t\right. \\
& \left.\quad+\left(1+\beta_{2}^{*}\right) \bar{\sigma}^{*}(t) d B_{t}^{1}-\eta_{t} d W_{t}\right\}, \quad t \in D^{C} .
\end{aligned}
$$

Let $X^{\pi, x}(t)$ denote the total wealth at time $t$, and $\pi(t)$ represents the proportion of the wealth invested in project $\mathrm{B}$; then $(1-\pi(t)) X^{\pi, x}(t)$ is the amount invested in project A. Taking inflation into consideration, both of them are calculated by its actual value. So we have

$$
\begin{aligned}
d X^{\pi, x}(t)= & \pi(t) X^{\pi, x}(t) \frac{d \Lambda^{*}(t)}{\Lambda^{*}(t)} \\
& +(1-\pi(t)) X^{\pi, x}(t) \frac{d \Lambda(t)}{\Lambda(t)}, \quad t \in D, \\
d X^{\pi, x}(t)= & \pi(t) X^{\pi, x}(t) \frac{d \bar{\Lambda}^{*}(t)}{\bar{\Lambda}^{*}(t)} \\
& +(1-\pi(t)) X^{\pi, x}(t) \frac{d \bar{\Lambda}(t)}{\bar{\Lambda}(t)}, \quad t \in D .
\end{aligned}
$$

Substituting $\Lambda_{t}, \Lambda^{*}(t), \bar{\Lambda}_{t}$, and $\bar{\Lambda}_{t}^{*}$ parts by (12) and (13), respectively, we get that, for an investor whose initial wealth is $x>0$, the total wealth at time $t$ in inflation case satisfies

$$
\begin{aligned}
& d X^{\pi, x}(t) \\
& =X^{\pi, x}(t)\left[\pi ( t ) \left(f^{*}(t)-\theta_{t}+\left(\eta_{t}\right)^{2}\right.\right. \\
& \left.\quad-\left(1+\beta_{1}^{*}\right) \rho_{2} \sigma^{*}(t) \eta_{t}\right)+(1-\pi(t)) \\
& \left.\quad \times\left(f(t)-\theta_{t}+\left(\eta_{t}\right)^{2}-\left(1+\beta_{1}\right) \rho_{1} \sigma(t) \eta_{t}\right)\right] d t \\
& \quad-c_{t}^{1} d t-\eta_{t} X^{\pi, x}(t) d W_{t} \\
& +\pi(t) X^{\pi, x}(t)\left(1+\beta_{1}^{*}\right) \sigma^{*}(t) d B_{t}^{2} \\
& +(1-\pi(t)) X^{\pi, x}(t)\left(1+\beta_{1}\right) \sigma(t) d B_{t}^{1}, \quad t \in D, \\
& \left.d X^{\pi, x}(t) \quad-\left(1+\beta_{2}^{*}\right) \rho_{2} \bar{\sigma}^{*}(t) \eta_{t}\right)+(1-\pi(t)) \\
& =X^{\pi, x}(t)\left[\pi ( t ) \left(\bar{f}^{*}(t)-\theta_{t}+\left(\eta_{t}\right)^{2}\right.\right. \\
& \left.\quad \times\left(\bar{f}(t)-\theta_{t}+\left(\eta_{t}\right)^{2}-\left(1+\beta_{2}\right) \rho_{1} \bar{\sigma}(t) \eta_{t}\right)\right] d t \\
& +c_{t}^{2} d t-\eta_{t} X^{\pi, x}(t) d W_{t} \\
& +\pi(t) X^{\pi, x}(t)\left(1+\beta_{2}^{*}\right) \bar{\sigma}^{*}(t) d B_{t}^{2} \\
& +(1-\pi(t)) X^{\pi, x}(t)\left(1+\beta_{2}\right) \bar{\sigma}(t) d B_{t}^{1}, \quad t \in D^{C} .
\end{aligned}
$$

In the daytime, the investor can choose investment with proportion $\pi$ and consumption rate $c^{1}$ to maximize his wealth, but at the nighttime, he cannot change his portfolio and only can choose a different consumption rate $c^{2}$; the portfolio at the nighttime stays the same as the optimal portfolio in the daytime. So the investor wants to maximize the following utility of wealth by choosing his investment strategy $\pi$ and consumption rate $c^{1}$ and $c^{2}$. Let $J^{1}(X)$ the value of wealth $X$ starting the daytime and $J^{2}(X)$ be the value of the wealth $X$ starting the nighttime. The whole investment duration can be divided into $n$ days, that is, $n T$ daytime duration and $n N$ the nighttime duration. Consider

$$
J^{1}\left(X_{0}\right)=\max _{\left(\pi, c^{1}\right)} \mathbb{E}\left[\int_{0}^{T} e^{-\gamma t} U\left(c^{1}(t)\right) d t+e^{-\gamma T} J^{2}\left(X_{T}\right)\right],
$$

$J^{2}\left(X_{T}\right)$

$$
\begin{aligned}
& =\max _{c^{2}} \mathbb{E}_{T}\left[\int_{T}^{T+N} e^{-\gamma(t-T)} U\left(c^{2}(t)\right) d t+e^{-\gamma N} J^{1}\left(X_{T+N}\right)\right], \\
& \vdots
\end{aligned}
$$

$$
\begin{aligned}
& J^{1}\left(X_{(n-1)(T+N)}\right) \\
& =\max _{\left(\pi, c^{1}\right)} \mathbb{E}_{(n-1)(T+N)} \\
& \times\left[\int_{(n-1)(T+N)}^{n T+(n-1) N} e^{-\gamma(t-(n-1)(T+N))} U\left(c^{1}(t)\right) d t\right. \\
& \left.\quad+e^{-\gamma T} J^{2}\left(X_{n T+(n-1) N}\right)\right] \\
& J^{2}\left(X_{n T+(n-1) N}\right) \\
& =\max _{c^{2}} \mathbb{E}_{n T+(n-1) N} \\
& \times\left[\int_{n T+(n-1) N}^{n(T+N)} e^{-\gamma(t-n T+(n-1) N)} U\left(c^{2}(t)\right) d t\right. \\
& \left.\quad+e^{-\gamma N} h\left(X_{n(T+N)}\right)\right] .
\end{aligned}
$$

The optimal problem (15)-(19) can be solved by the classical methods, such as Pontryagin's maximum principle (cf., e.g., Yong and Zhou [10], Wang et al. [11], Wang and Wu [12], etc.) which gives the necessary conditions for the optimal strategies and dynamic programming principle (cf., e.g., Yong and Zhou [10]). In the following section, we will use a simple method, called construction method, to give an explicit solution to this problem in a special HARA case and give the economic analysis.

\section{HARA Case: Optimal Solutions and Economic Analysis}

In this section, we want to use the construction method to get the explicit optimal strategy in the special Hyperbolic 
Absolute Risk Aversion (HARA) case. This method can also be found when solving the linear quadratic (LQ) optimal control problem. It is often along with the dynamic programming principle; see Yong and Zhou [10], for example. For simplicity, we only consider the case where the entire time interval is $T+N, T$ is the duration of the daytime, and $N$ is the duration of the nighttime. For general case, the whole interval is $n(T+N)$, and we can get the explicit result by repeating the procedure with the same method.

Let

$$
\begin{gathered}
J^{1}\left(X_{0}\right)=\max _{\left(\pi, c_{t}^{1}\right)} \mathbb{E}\left[\int_{0}^{T} e^{-\gamma t} \frac{\left(c_{t}^{1}\right)^{1-R}}{1-R} d t+e^{-\gamma T} J^{2}\left(X_{T}\right)\right], \\
J^{2}\left(X_{T}\right)=\max _{c_{t}^{2}} \mathbb{E}\left[\int_{T}^{T+N} e^{-\gamma t} \frac{\left(c_{t}^{2}\right)^{1-R}}{1-R} d t+e^{-\gamma N} K \frac{X_{T+N}^{1-R}}{1-R}\right] .
\end{gathered}
$$

Here $\gamma$ and $R$ are constants, where $\gamma>0, R \in(0,1)$. We try to get explicit optimal decision $\pi$, consumption rates $c^{1}, c^{2}$, and the optimal value function in this case.

Theorem 1. Under all the above assumptions, the optimal strategies to the optimal portfolio choice problem (18), (19), (20), and (21) for the specific HARA case are given by

$$
\begin{gathered}
J^{1}\left(X_{0}\right)=\frac{1}{1-R}\left(X_{0}^{\pi, x}\right)^{1-R} \Theta_{0}, \\
J^{2}\left(X_{T}\right)=\frac{1}{1-R}\left(X_{T}^{\pi, x}\right)^{1-R} \Theta_{T}, \\
\pi^{*}(t)=\frac{\Delta^{u}}{\Delta^{d}}, \quad t \in[0, T+N], \\
\left(c_{t}^{1}\right)^{*}=(1-R)^{-1 / R}\left(\Theta_{t}\right)^{-1 / R} X_{t}^{\pi, x}, \quad t \in[0, T], \\
\left(c_{t}^{2}\right)^{*}=(1-R)^{-1 / R}\left(\Theta_{t}\right)^{-1 / R} X_{t}^{\pi, x}, \quad t \in[T, T+N],
\end{gathered}
$$

where $\Theta_{t}$ satisfies ODEs (27), (41), and $\Delta^{u}, \Delta^{d}$ are denoted by (37), (38), respectively.

Proof. We first consider the optimal problem (21) at nighttime. During the night, the investor cannot change his portfolio. His consumption $c^{2}$ is the only activity that varies. So, the wealth equation in the duration $[T, T+N]$ is,

$$
\begin{gathered}
d X^{\pi, x}(t) \\
=X^{\pi, x}(t)\left[\pi ^ { * } ( t ) \left(\bar{f}^{*}(t)-\theta_{t}+\left(\eta_{t}\right)^{2}\right.\right. \\
\left.-\left(1+\beta_{2}^{*}\right) \rho_{2} \bar{\sigma}^{*}(t) \eta_{t}\right) \\
+\left(1-\pi^{*}(t)\right)\left(\bar{f}(t)-\theta_{t}+\left(\eta_{t}\right)^{2}\right. \\
\left.\left.\quad-\left(1+\beta_{2}\right) \rho_{1} \bar{\sigma}(t) \eta_{t}\right)\right] d t \\
-c_{t}^{2} d t-\eta_{t} X^{\pi, x}(t) d W_{t}
\end{gathered}
$$

$$
\begin{array}{r}
+\pi^{*}(t) X^{\pi, x}(t)\left(1+\beta_{2}^{*}\right) \bar{\sigma}^{*}(t) d B_{t}^{2} \\
+\left(1-\pi^{*}(t)\right) X^{\pi, x}(t)\left(1+\beta_{2}\right) \times \bar{\sigma}(t) d B_{t}^{1}, \\
t \in[T, T+N] .
\end{array}
$$

Here $\pi^{*}$ is the optimal portfolio in the daytime, and we will solve it afterward.

We let $\Theta_{t}$ be one nonnegative deterministic continuous function whose dynamic will be given later. Applying Ito's formula to $\left(e^{-\gamma(t-T)} /(1-R)\right)\left(X^{\pi, x}(t)\right)^{1-R} \Theta_{t}$ from $T$ to $T+N$, we have

$$
\begin{aligned}
& \mathbb{E}_{T}\left[\frac{e^{-\gamma N}}{1-R}\left(X_{T+N}^{\pi, x}\right)^{1-R} \Theta_{T+N}\right] \\
& =\frac{1}{1-R}\left(X_{T}^{\pi, x}\right)^{1-R} \Theta_{T} \\
& +\mathbb{E}_{T} \int_{T}^{T+N}\left\{\Theta _ { t } \left[-\gamma \frac{e^{-\gamma(t-T)}}{1-R}\left(X_{t}^{\pi, x}\right)^{1-R}\right.\right. \\
& +\frac{e^{-\gamma(t-T)}}{1-R}\left(X_{t}^{\pi, x}\right)^{1-R}(1-R) \\
& \times\left[\pi ^ { * } ( t ) \left(\bar{f}^{*}(t)-\theta_{t}+\left(\eta_{t}\right)^{2}\right.\right. \\
& \left.-\left(1+\beta_{2}^{*}\right) \rho_{2} \bar{\sigma}^{*}(t) \eta_{t}\right) \\
& +\left(1-\pi^{*}(t)\right)\left(\bar{f}(t)-\theta_{t}+\left(\eta_{t}\right)^{2}\right. \\
& \left.\left.-\left(1+\beta_{2}\right) \rho_{1} \bar{\sigma}(t) \eta_{t}\right)\right] \\
& -\frac{1}{2} R(1-R) e^{-\gamma(t-T)}\left(X_{t}^{\pi, x}\right)^{1-R} \\
& \times\left[\eta(t)^{2}+\left(\pi^{*}(t)\right)^{2}\left(1+\beta_{2}^{*}\right)^{2}\left(\bar{\sigma}^{*}(t)\right)^{2}\right. \\
& \left.+\left(1-\pi^{*}(t)\right)^{2}\left(1+\beta_{2}\right)^{2}(\bar{\sigma}(t))^{2}\right] \\
& -R(1-R) e^{-\gamma(t-T)}\left(X_{t}^{\pi, x}\right)^{1-R} \\
& \times\left[-\eta(t)\left(\pi^{*}(t)\right)\left(1+\beta_{2}^{*}\right) \bar{\sigma}^{*}(t) \rho_{2}\right. \\
& -\eta(t)\left(1-\pi^{*}(t)\right)\left(1+\beta_{2}\right) \bar{\sigma}(t) \rho_{1} \\
& +\pi^{*}(t)\left(1-\pi^{*}(t)\right)\left(1+\beta_{2}^{*}\right) \\
& \left.\times\left(1+\beta_{2}\right) \bar{\sigma}(t) \bar{\sigma}^{*}(t) \rho_{1,2}\right] \\
& \left.-\frac{e^{-\gamma(t-T)}}{1-R}\left(X_{t}^{\pi, x}\right)^{-R}(1-R) c_{t}^{2}\right] \\
& \left.+\frac{e^{-\gamma(t-T)}}{1-R}\left(X_{t}^{\pi, x}\right)^{1-R} \dot{\Theta}_{t}\right\} d t .
\end{aligned}
$$

So we can write

$$
J^{2}\left(X_{T}\right)=\frac{1}{1-R}\left(X_{T}^{\pi, x}\right)^{1-R} \Theta_{T}+I+I I+I I I,
$$


where

$$
\begin{aligned}
& I=\max _{c_{t}^{2}} \mathbb{E}_{T} \int_{T}^{T+N} \frac{e^{-\gamma(t-T)}}{1-R}\left[\left(c_{t}^{2}\right)^{1-R}-(1-R)\left(X_{t}^{\pi, x}\right)^{-R} \Theta_{t} c_{t}^{2}\right. \\
& \left.-R\left(X_{t}^{\pi, x}\right)^{1-R} \Theta_{t}^{1-(1 / R)}\right] d t, \\
& I I=\max _{c_{t}^{2}} \mathbb{E}_{T} \\
& \times \int_{T}^{T+N} \frac{e^{-\gamma(t-T)}}{1-R}\left(X_{t}^{\pi, x}\right)^{1-R} \\
& \times\left\{\dot{\Theta}_{t}+\Theta_{t}\right. \\
& \times\left[-\gamma+(1-R) \pi^{*}(t)\right. \\
& \times\left[\bar{f}^{*}(t)-\theta_{t}+\left(\eta_{t}\right)^{2}-\left(1+\beta_{2}^{*}\right) \rho_{2} \bar{\sigma}^{*}(t) \eta_{t}\right] \\
& +(1-R)\left(1-\pi^{*}(t)\right) \\
& \times\left[\bar{f}(t)-\theta_{t}+\left(\eta_{t}\right)^{2}-\left(1+\beta_{2}\right) \rho_{1} \bar{\sigma}(t) \eta_{t}\right] \\
& -\frac{1}{2} R(1-R) \\
& \times\left[\eta(t)^{2}+\left(\pi^{*}(t)\right)^{2}\left(1+\beta_{2}^{*}\right)^{2}\left(\bar{\sigma}^{*}(t)\right)^{2}\right. \\
& \left.+\left(1-\pi^{*}(t)\right)^{2}\left(1+\beta_{2}\right)^{2}(\bar{\sigma}(t))^{2}\right] \\
& -R(1-R)\left[-\eta(t)\left(\pi^{*}(t)\right)\left(1+\beta_{2}^{*}\right) \bar{\sigma}^{*}(t) \rho_{2}\right. \\
& -\eta(t)\left(1-\pi^{*}(t)\right)\left(1+\beta_{2}\right) \bar{\sigma}(t) \\
& \times \rho_{1}+\pi^{*}(t)\left(1-\pi^{*}(t)\right)\left(1+\beta_{2}^{*}\right) \\
& \left.\left.\times\left(1+\beta_{2}\right) \bar{\sigma}(t) \bar{\sigma}^{*}(t) \rho_{1,2}\right]\right] \\
& \left.+R \Theta_{t}^{1-(1 / R)}\right\} d t, \\
& I I I=\max _{c^{2}} \mathbb{E}_{T}\left[\frac{e^{-\gamma N}}{1-R}\left(X_{T+N}^{\pi, x}\right)^{1-R}\left(K-\Theta_{T+N}\right)\right] .
\end{aligned}
$$

Now we let $\Theta_{t}$ be nonnegative and the solution of the following ordinary differential equation:

$$
\begin{gathered}
-\dot{\Theta}_{t}=\bar{M} \Theta_{t}+R \Theta_{t}^{1-(1 / R)}, \quad t \in[T, T+N], \\
\Theta_{T+N}=K .
\end{gathered}
$$

Here

$$
\begin{aligned}
\bar{M}= & -\gamma+(1-R) \pi^{*}(t) \\
& \times\left[\bar{f}^{*}(t)-\theta_{t}+\left(\eta_{t}\right)^{2}-\left(1+\beta_{2}^{*}\right) \rho_{2} \bar{\sigma}^{*}(t) \eta_{t}\right] \\
& +(1-R)\left(1-\pi^{*}(t)\right) \\
& \times\left[\bar{f}(t)-\theta_{t}+\left(\eta_{t}\right)^{2}-\left(1+\beta_{2}\right) \rho_{1} \bar{\sigma}(t) \eta_{t}\right]
\end{aligned}
$$

$$
\begin{aligned}
-\frac{1}{2} R(1-R)[ & \eta(t)^{2}+\left(\pi^{*}(t)\right)^{2}\left(1+\beta_{2}^{*}\right)^{2}\left(\bar{\sigma}^{*}(t)\right)^{2} \\
& \left.+\left(1-\pi^{*}(t)\right)^{2}\left(1+\beta_{2}\right)^{2}(\bar{\sigma}(t))^{2}\right] \\
-R(1-R)[ & -\eta(t)\left(\pi^{*}(t)\right)\left(1+\beta_{2}^{*}\right) \bar{\sigma}^{*}(t) \rho_{2} \\
- & \eta(t)\left(1-\pi^{*}(t)\right)\left(1+\beta_{2}\right) \bar{\sigma}(t) \rho_{1} \\
& +\pi^{*}(t)\left(1-\pi^{*}(t)\right)\left(1+\beta_{2}^{*}\right) \\
& \left.\times\left(1+\beta_{2}\right) \bar{\sigma}(t) \bar{\sigma}^{*}(t) \rho_{1,2}\right] .
\end{aligned}
$$

So

$$
\begin{array}{r}
\Theta_{t}=e^{\bar{M}(T+N-t)}\left[K+\int_{t}^{T+N} e^{-(1 / R) \bar{M}(T+N-s)} d s\right]^{R}, \\
t \in[T, T+N], \\
\Theta_{T}=e^{\bar{M} N}\left[K+\int_{T}^{T+N} e^{-(1 / R) \bar{M}(T+N-s)} d s\right]^{R} .
\end{array}
$$

We can easily get $I I=0, I I I=0$.

If we take

$$
\left(c_{t}^{2}\right)^{*}=(1-R)^{-1 / R}\left(\Theta_{t}\right)^{-1 / R} X_{t}^{\pi, x}, \quad t \in[T, T+N],
$$

then we can check that $I$ attains its maximum at point $\left(C^{2}\right)^{*}$ and $I=0$. So

$$
J^{2}\left(X_{T}\right)=\frac{1}{1-R}\left(X_{T}^{\pi, x}\right)^{1-R} \Theta_{T} .
$$

And then applying Ito's formula to $\left(e^{-\gamma t} /(1-R)\right)\left(X_{t}^{\pi, x}\right)^{1-R} \Theta_{t}$ from 0 to $T$ and taking expectation on both sides, we have

$$
\begin{aligned}
& \mathbb{E}\left[\frac{e^{-\gamma T}}{1-R}\left(X_{T}^{\pi, x}\right)^{1-R} \Theta_{T}\right] \\
& =\frac{1}{1-R}\left(X_{0}^{\pi, x}\right)^{1-R} \Theta_{0} \\
& +\mathbb{E} \int_{0}^{T}\left\{\Theta _ { t } \left[-\gamma \frac{e^{-\gamma t}}{1-R}\left(X_{t}^{\pi, x}\right)^{1-R}+\frac{e^{-\gamma t}}{1-R}\left(X_{t}^{\pi, x}\right)^{1-R}\right.\right. \\
& \times(1-R)\left[\pi ( t ) \left(f^{*}(t)-\theta_{t}+\left(\eta_{t}\right)^{2}\right.\right.
\end{aligned}
$$$$
\left.-\left(1+\beta_{1}^{*}\right) \rho_{2} \sigma^{*}(t) \eta_{t}\right)
$$$$
+(1-\pi(t))
$$$$
\times\left(f(t)-\theta_{t}+\left(\eta_{t}\right)^{2}\right.
$$$$
\left.\left.-\left(1+\beta_{1}\right) \rho_{1} \sigma(t) \eta_{t}\right)\right]
$$

$$
\begin{aligned}
& \quad-\frac{1}{2} R e^{-\gamma t}\left(X_{t}^{\pi, x}\right)^{1-R} \\
& \times\left[\left(\eta_{t}\right)^{2}+(\pi(t))^{2}\left(1+\beta_{1}^{*}\right)^{2}\left(\sigma^{*}(t)\right)^{2}\right. \\
& \left.\quad+(1-\pi(t))^{2}\left(1+\beta_{1}\right)^{2}(\sigma(t))^{2}\right] \\
& \quad-R e^{-\gamma t}\left(X_{t}^{\pi, x}\right)^{1-R}
\end{aligned}
$$




$$
\begin{aligned}
& \times[-\eta_{t} \pi(t)\left(1+\beta_{1}^{*}\right) \sigma^{*}(t) \rho_{2} \\
&-\eta_{t}(1-\pi(t))\left(1+\beta_{1}\right) \sigma(t) \rho_{1} \\
&+\pi(t)(1-\pi(t))\left(1+\beta_{1}^{*}\right) \\
&\left.\times\left(1+\beta_{1}\right) \sigma(t) \sigma^{*}(t) \rho_{1,2}\right] \\
&\left.-\frac{e^{-\gamma t}}{1-R}\left(X_{t}^{\pi, x}\right)^{-R}(1-R) c_{t}^{1}\right] \\
&\left.+\frac{e^{-\gamma t}}{1-R}\left(X_{t}^{\pi, x}\right)^{1-R} \dot{\Theta}_{t}\right\} d t .
\end{aligned}
$$

So we can write

$$
J^{1}\left(X_{0}\right)=\frac{1}{1-R}\left(X_{0}^{\pi, x}\right)^{1-R} \Theta_{0}+I V+V+V I,
$$

where

$$
\begin{gathered}
I V=\max _{\left(\pi, c_{t}^{1}\right)} \mathbb{E} \int_{0}^{T} \frac{e^{-\gamma(t-T)}}{1-R}\left[\left(c_{t}^{1}\right)^{1-R}-(1-R)\left(X_{t}^{\pi, x}\right)^{-R} \Theta_{t} c_{t}^{1}\right. \\
\left.-R X_{t}^{1-R} \Theta_{t}^{1-(1 / R)}\right] d t, \\
V=\max _{\left(\pi, c_{t}^{1}\right)} \mathbb{E} \int_{0}^{T} e^{-\gamma t}\left(X_{t}^{\pi, x}\right)^{1-R} \Theta_{t} L(\pi(t)) d t .
\end{gathered}
$$

Here

$$
\begin{gathered}
L(x)=x\left(f^{*}(t)-f(t)+(1-R) \eta_{t}\right. \\
\times\left[\left(1+\beta_{1}\right) \rho_{1} \sigma(t)-\left(1+\beta_{1}^{*}\right) \rho_{2} \sigma^{*}(t)\right] \\
+R\left(1+\beta_{1}\right)^{2}(\sigma(t))^{2} \\
\left.-R\left(1+\beta_{1}^{*}\right)\left(1+\beta_{1}\right) \sigma(t) \sigma^{*}(t) \rho_{1,2}\right) \\
-\frac{1}{2} x^{2}\left(R\left[\left(1+\beta_{1}^{*}\right)^{2}\left(\sigma^{*}(t)\right)^{2}+\left(1+\beta_{1}\right)^{2}(\sigma(t))^{2}\right]\right. \\
\left.\quad-2 R\left(1+\beta_{1}\right)\left(1+\beta_{1}^{*}\right) \sigma(t) \sigma^{*}(t) \rho_{1,2}\right)
\end{gathered}
$$$$
-\Delta
$$

$$
\begin{aligned}
\Delta^{u}= & f^{*}(t)-f(t)+(1-R) \eta_{t} \\
& \times\left[\left(1+\beta_{1}\right) \rho_{1} \sigma(t)-\left(1+\beta_{1}^{*}\right) \rho_{2} \sigma^{*}(t)\right] \\
& +R\left(1+\beta_{1}\right)^{2}(\sigma(t))^{2}-R\left(1+\beta_{1}^{*}\right) \\
& \times\left(1+\beta_{1}\right) \sigma(t) \sigma^{*}(t) \rho_{1,2}, \\
\Delta^{d}= & R\left[\left(1+\beta_{1}^{*}\right)^{2}\left(\sigma^{*}(t)\right)^{2}+\left(1+\beta_{1}\right)^{2}(\sigma(t))^{2}\right] \\
& -2 R\left(1+\beta_{1}\right)\left(1+\beta_{1}^{*}\right) \sigma(t) \sigma^{*}(t) \rho_{1,2},
\end{aligned}
$$

$$
\begin{aligned}
V I=\max _{\left(\pi, c_{t}^{1}\right)} \mathbb{E} \int_{0}^{T} \frac{e^{-\gamma t}}{1-R} X_{t}^{1-R} & \\
\times\left\{\dot{\Theta}_{t}+\Theta_{t}[\right. & -\gamma+(1-R) \\
& \times\left[f(t)-\theta_{t}+\left(\eta_{t}\right)^{2}\right. \\
& \left.-\left(1+\beta_{1}\right) \rho_{1} \sigma(t) \eta_{t}\right] \\
& -\frac{1}{2} R(1-R) \\
& \times\left[\eta(t)^{2}+\left(1+\beta_{1}\right)^{2}(\sigma(t))^{2}\right] \\
& +R(1-R) \eta(t)\left(1+\beta_{1}\right) \sigma(t) \rho_{1} \\
& \left.+(1-R) \Delta]+R \Theta_{t}^{1-(1 / R)}\right\} .
\end{aligned}
$$

If we take

$$
\pi^{*}(t)=\frac{\Delta^{u}}{\Delta^{d}},
$$

it is easy to check that $L^{\prime}\left(\pi^{*}(t)\right)=0$ and $L^{\prime \prime}\left(\pi^{*}\right)<0$. Thus the function $L(\pi)$ attains its maximum at point $\pi^{*}$ and $L\left(\pi^{*}\right)=0$, $V=0$. Now we let $P_{t}$ be the solution of the following ODE:

$$
-\dot{\Theta}_{t}=M \Theta_{t}+R \Theta_{t}^{1-(1 / R)}, \quad t \in[0, T],
$$

$$
\Theta_{T}=e^{\bar{M} N}\left[K+\int_{T}^{T+N} e^{-(1 / R) \bar{M}(T+N-s)} d s\right]^{R} .
$$

Here

$$
\begin{aligned}
M= & -\gamma+(1-R)\left[f(t)-\theta_{t}+\left(\eta_{t}\right)^{2}-\left(1+\beta_{1}\right) \rho_{1} \sigma(t) \eta_{t}\right] \\
& -\frac{1}{2} R(1-R)\left[\eta(t)^{2}+\left(1+\beta_{1}\right)^{2}(\sigma(t))^{2}\right] \\
& +R(1-R) \eta(t)\left(1+\beta_{1}\right) \sigma(t) \rho_{1}+(1-R) \Delta .
\end{aligned}
$$

We have

$$
\begin{gathered}
P_{t}=e^{M(T-t)}\left[P_{T}^{1 / R}+\int_{t}^{T} e^{-(1 / R) M(T-s)} d s\right]^{R}, \quad t \in[0, T], \\
P_{0}=e^{M T}\left[P_{T}^{1 / R}+\int_{0}^{T} e^{-(1 / R) M(T-s)} d s\right]^{R} .
\end{gathered}
$$

Then $V I=0$.

Let

$$
\left(c_{t}^{1}\right)^{*}=(1-R)^{-1 / R}\left(\Theta_{t}\right)^{-1 / R} X_{t}^{\pi, x}, \quad t \in[0, T] .
$$

One can check that $I V$ attains its maximum at point $\left(c_{t}^{1}\right)^{*}$, $I V=0$. Then the optimal value function is

$$
J^{1}\left(X_{0}\right)=\frac{1}{1-R}\left(X_{0}^{\pi, x}\right)^{1-R} \Theta_{0} .
$$


Remark 2. In HARA case, we can get from simple calculus that the Pratt-Arrow measure of relative aversion $A=R, R \in$ $(0,1)$.

So the constant $R$ can indicate the investor's attitude to the risk in the investment.

Once knowing the amount of our wealth, we can make a decision on the strategy of the investment according to formula (22).

\section{Simulation Results}

From the history of price data in the market, we can use statistical method to estimate the parameters in the model. Now let us give a simulating example. In this example, let the coefficients be constants for simplicity, and we only consider the choice at initial time $t=0$.

Example 3. We take the following parameters depending on the situation of the real market. Choose $S_{0}=30, S_{0}^{*}=50$, $P_{0}=15, S_{0}^{*}=26, \beta_{1}=-0.28, \beta_{2}=-0.1, \alpha_{S}=1.5, \alpha_{S}^{*}=1.2$, $\alpha_{p}=1.4, \alpha_{p}^{*}=1.1, \gamma=0.6, \rho_{1}=0.25, \rho_{2}=-0.1, \rho_{1,2}=-0.3$, $\eta=0.1, T=2$, and $R=0.3$.

From the formula (22), we know that the optimal portfolio $\pi$ is a 2 -dimensional function with the volatility parameters $\sigma$ and $\pi^{*}$, and we cannot get the explicitly monotone relationship. Next we will see the relationship of them by the 3D figure. For fixed $\sigma=0.3$ (see Figure 1), we can get the relationship between the optimal proportion $\pi$ and the volatility of the project $\mathrm{B} \sigma^{*}$. Here $\pi$ decreases down to 0 . That means the high volatility leads to more capital investment in project A and less investment in project B. Taking $\sigma=0.69$ for example, here $\pi^{*}=0.5616$, that is, the invest's optimal choice is to invest almost half of his wealth to project $B$. When $\sigma=0.84, \pi^{*}=0.273$; that is, the investor would only devote about thirty percent of his wealth to the corporate project and invest the rest in project $\mathrm{A}$.

In Figure 2, the curve goes just as we have expected. From the figure, we can easily get the relationship between the optimal proportion $\pi^{*}$ and the volatility of the production $\sigma$ and $\pi^{*}$. Taking $\sigma=0.54, \sigma^{*}=0.32$, for example, here $\pi^{*}=$ 0.5323 . In our model, $\pi(t)$ represents the proportion of the wealth invested in project $B$. The volatility of project $B$ is bigger than project $\mathrm{A}$. Then the investor will invest less wealth to project $\mathrm{B}$.

Next for fixed $\sigma^{*}=0.5$, we will discuss the relationship between the portfolio and the volatility $\sigma$ and inflation ratio. In Figure 3, the curve goes just as we expect. Let us take the two points $(0.414,0.097,1.207)$ and $(0.414,0.09,0.4144)$ for example. From the figure, for fixed $\sigma$, we know that when the inflation ratio decreases, the investor will invest more money to project A.

\section{Conclusions}

In this paper, we present the model of corporate optimal investment with consideration of the influence of inflation and the difference between the daytime and nighttime.

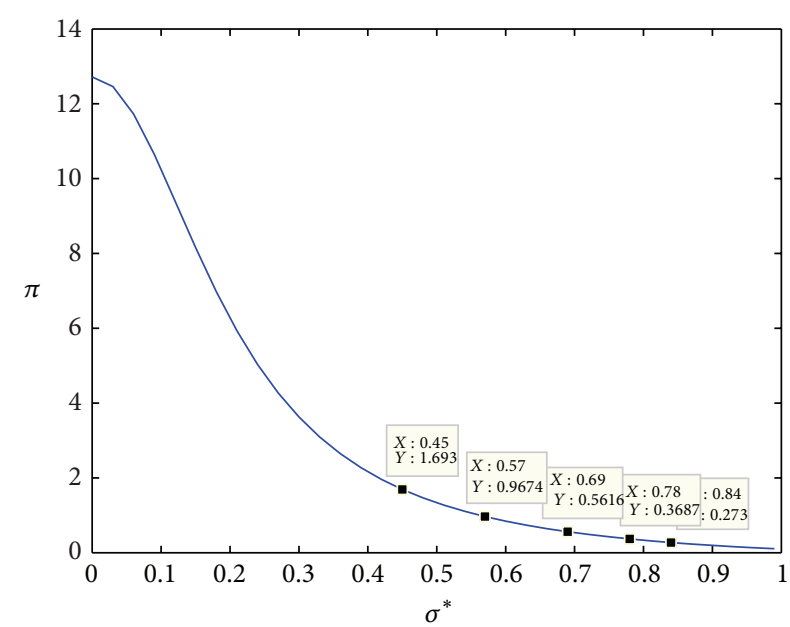

FIGURE 1: Relationship between the volatility of project B $\sigma^{*}$ and the optimal proportion $\pi^{*}$.

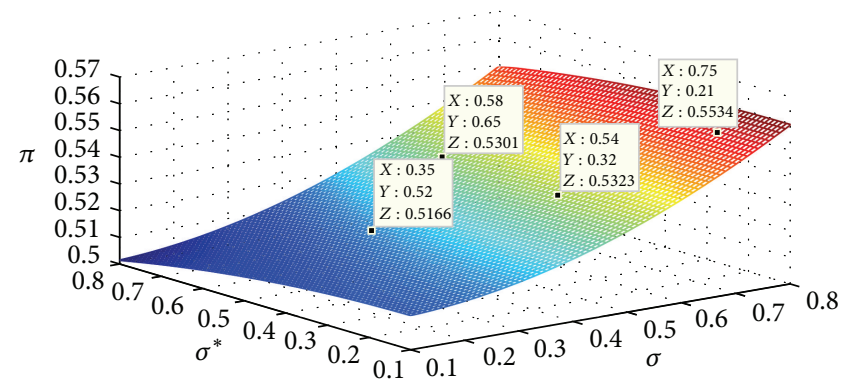

FIGURE 2: Relationship between the volatility of the project $\sigma, \sigma^{*}$ and the optimal proportion $\pi^{*}$.

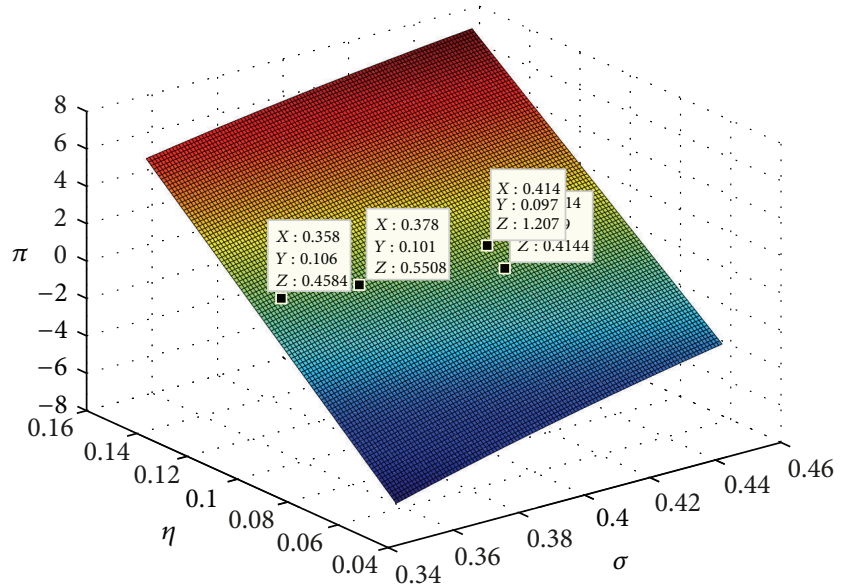

FIGURE 3: Relationship between the volatility of project A $\sigma$, the inflation ratio $\eta$, and the optimal proportion $\pi^{*}$.

From Theorem 1, we know that the classical dynamical programming principle still holds for this optimal problem. The optimal investment and consumption choice have also been achieved by solving the corresponding H-J-B equation, and the economic analysis was then given for that. For a typical HARA utility function case, we got the explicit 
optimal investment and consumption strategy and then some simulation results illustrating the influence of market's volatility parameters to the optimal choice. In addition, there are several interesting problems about the underlying topic, such as the corresponding convex restriction problem and the partial information problem which needs to use the Pontryagin's maximum principle under partial information (Wang and $\mathrm{Wu}[12,13]$, etc.). Since these problems are more consistent with the practical situation in real market, we will furthermore investigate them in further work and then desire to solve some practical optimization investment problems in financial market.

\section{Acknowledgments}

Zongyuan Huang is supported in part by Chinese NSF Grant nos. 11126208 and 61174092. Detao Zhang is supported in part by Independent Innovation Foundation of Shandong University (2011GN018).

\section{References}

[1] R. Merton, "An equilibrium market model with incomplete information," Journal of Finance, vol. 42, no. 3, pp. 483-510, 1987.

[2] D. Duffie, Dynamic Asset Pricing Theory, Princeton University Press, Princeton, NJ, USA, 1992.

[3] I. Karatzas, "Optimization problems in the theory of continuous trading," SIAM Journal on Control and Optimization, vol. 27, no. 6, pp. 1221-1259, 1989.

[4] J. J. Choi, "Diversification, exchange risks and corporate international investment," Journal of International Business Studies, vol. 20, no. 1, pp. 145-155, 1989.

[5] M. Bellalah and Z. Wu, "A simple model of corporate international investment under incomplete information and taxes," Annals of Operations Research, vol. 165, no. 1, pp. 123-143, 2009.

[6] Z. Wu and L. Y. Zhang, "The corporate optimal portfolio and consumption choice problem in the real project with borrowing rate higher than deposit rate," Applied Mathematics and Computation, vol. 175, no. 2, pp. 1596-1608, 2006.

[7] M. Bellalah and Z. Wu, "A model for market closure and international portfolio management within incomplete information," International Journal of Theoretical and Applied Finance, vol. 5, pp. 479-495, 2002.

[8] Z. Y. Huang and Z. Wu, "One kind of corporate international optimal investment and consumption choice problem," in Proceedings of the 27th Chinese Control Conference (CCC '08), pp. 603-606, Kunming, China, July 2008.

[9] D. T. Zhang and T. Zhang, "Optimal portfolio of corporate investment and consumption under market closure," International Journal of Business, vol. 17, no. 1, pp. 25-38, 2012.

[10] J. M. Yong and X. Y. Zhou, Stochastic Controls: Hamiltonian Systems and HJB Equations, Springer, New York, NY, USA, 1999.

[11] G. Wang, Z. Wu, and J. Xiong, "Maximum principles for forward-backward stochastic control systems with correlated state and observation noises," SIAM Journal on Control and Optimization, vol. 51, no. 1, pp. 491-524, 2013.

[12] G. C. Wang and Z. Wu, "The maximum principles for stochastic recursive optimal control problems under partial information," IEEE Transactions on Automatic Control, vol. 54, no. 6, pp. 12301242, 2009.
[13] G. C. Wang and Z. Wu, "General maximum principles for partially observed risk-sensitive optimal control problems and applications to finance," Journal of Optimization Theory and Applications, vol. 141, no. 3, pp. 677-700, 2009. 


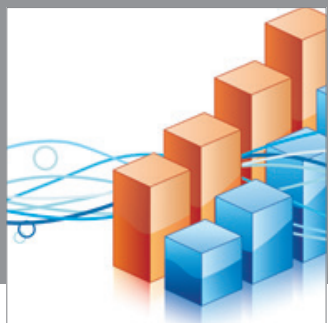

Advances in

Operations Research

mansans

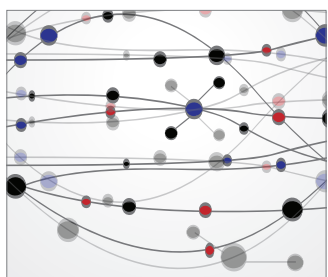

The Scientific World Journal
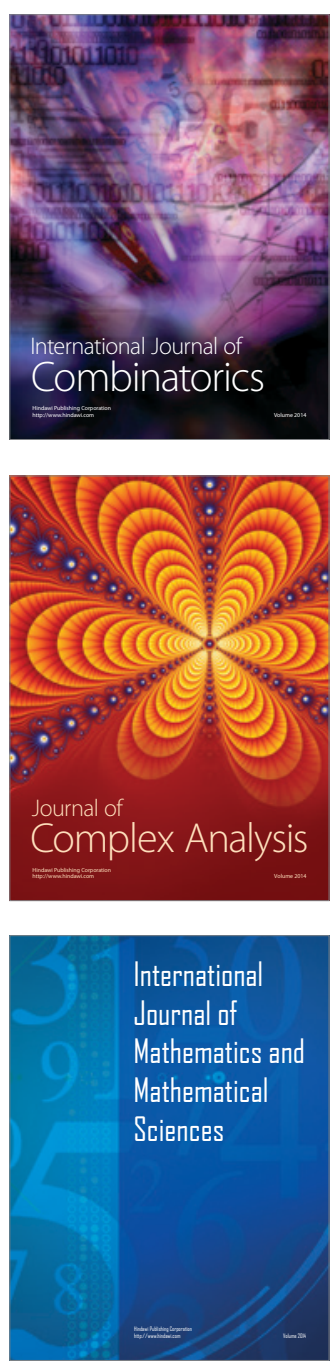
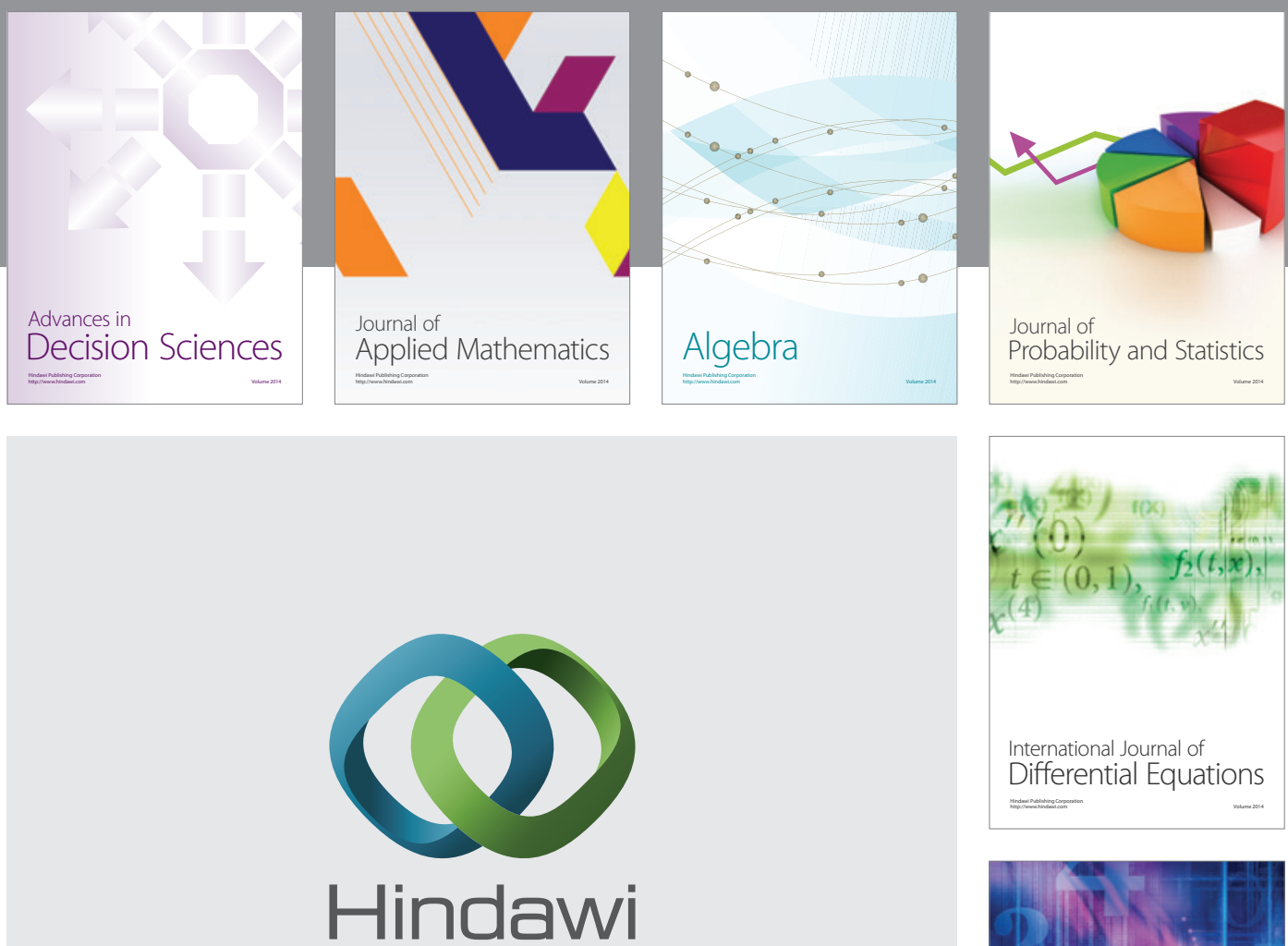

Submit your manuscripts at http://www.hindawi.com
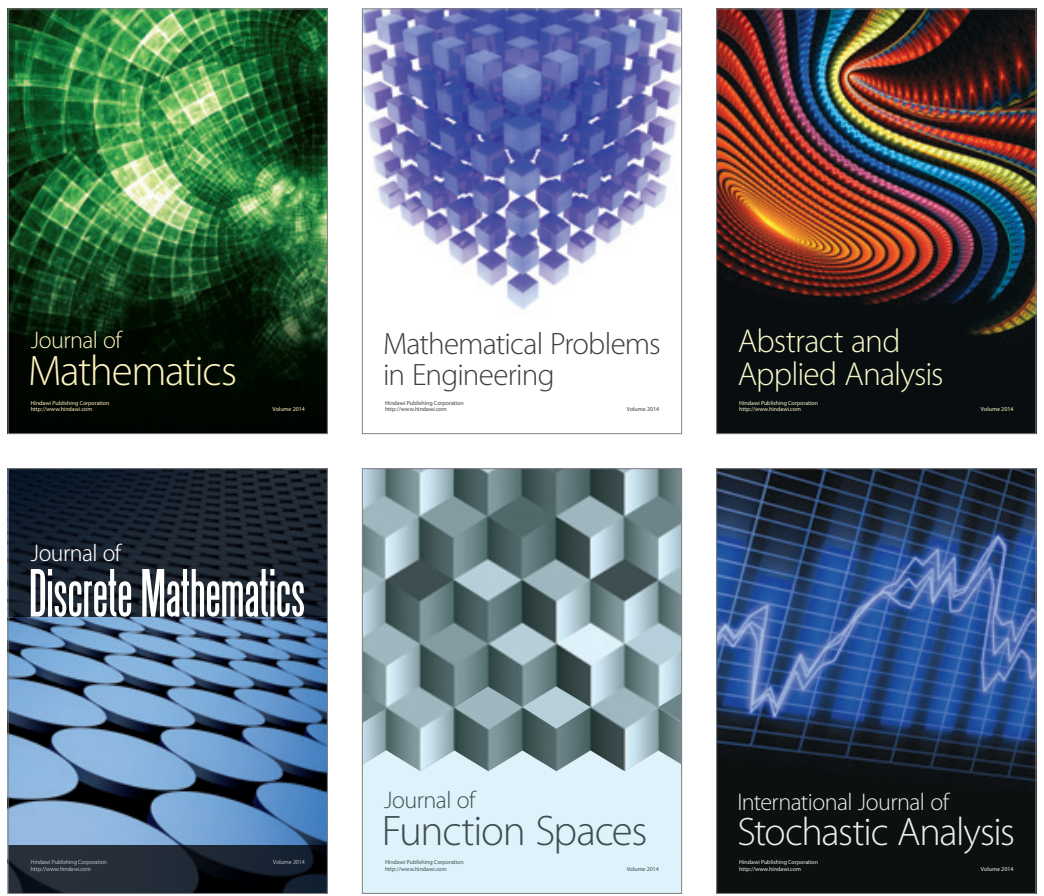

Journal of

Function Spaces

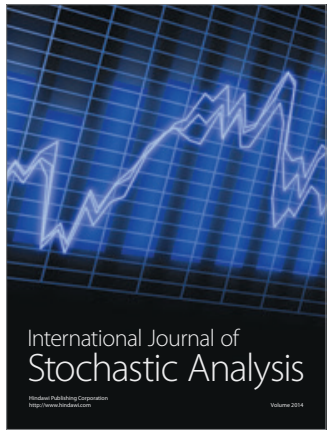

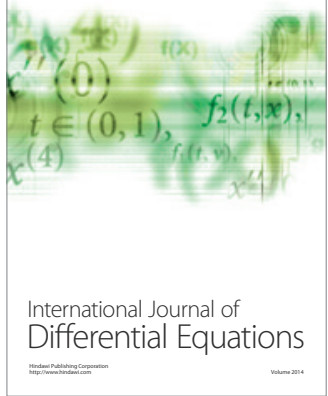
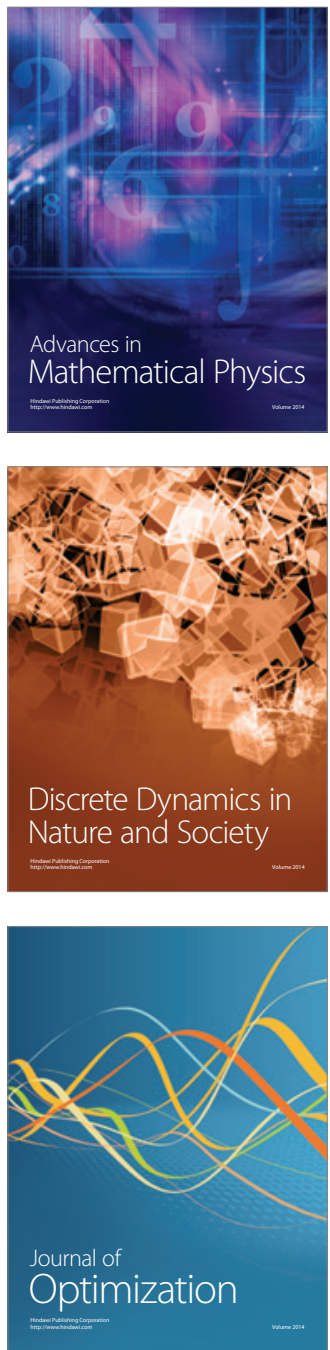